\title{
Una checklist per la gestione dei costi nel trattamento dell'HIV
}

Vasilisa Sazonov, Uros Urleb,, Albert I. Wertheimer§ articolo tratto dal Journal of Research in Pharmaceutical Economics, Vol. 10(1) 2000 E 2000 by The Haworth Press, Inc.

FARMECONOMIA NEL MONDO

\begin{abstract}
The human immunodeficiency virus (HIV) infection is currently one of the major health problems in the world. Since the first occurrence of the infection 17 years ago until today, many people have died of its devastating consequences and an enormous amount of health-related funds have been spent on the problem. The pandemic is still growing in some areas, and its negative impact on society is in-creasing. This is the main reason why scientists are trying to find the best way to reduce costs and still keep the same level of management or, in some ways, even improve it (e.g., new combination of drugs in one pill—fewer pills to be taken per day).

Since HIV infection has been. a fatal, severe, and widespread disease, a so-called global problem, very early in the course of the pandemic the idea of economic evaluation was raised.

Published studies mostly presented the antiretroviral drugs in the frames of pharmacoeconomic models or costs of HIV/AIDS according to development of the disease through longer time periods.

The cost sections that have to be considered in any pharmacoeconomic study of HIV management are briefly presented. Often the presented costs are confusing and could be misleading. Direct, indirect, and intangible costs are described.
\end{abstract}

Farmeconomia e percorsi terapeutici 2000; 1 (2): $91-96$

\section{INTRODUZIONE}

La questione globale della pandemia dell'AIDS

Il virus dell'HIV tipo 1 è causa della malattia mortale conosciuta come sindrome da immunodeficienza acquisita (AIDS). La malattia si è drammaticamente diffusa negli ultimi 17 anni, da quando la medicina ha osservato la prima comparsa negli omosessuali di una forma non spiegabile di polmonite Pneumocystis carinii e del sarcoma di Kaposi. I Centri per il Controllo delle Malattie (CDC) hanno ricevuto i primi rapporti nel 1981 e da quel momento il problema è divenuto di interesse globale in pochissimo tempo.

Oggi nel mondo 21,8 milioni di adulti e 830.000 bambini sono colpiti dall'infezione da HIV o hanno già sviluppato la sindrome dell'AIDS (1, 2). Le stime per il 1996 parlano di 29,4 milioni di persone colpite da HIV (26,8 milioni di adulti e 2,6 milioni di bambini), e occorre comunque precisare che in alcune zone il sistema di report è piuttosto arretrato, quindi i dati non sono attendibili in assoluto. Nel $1997 \mathrm{i}$ casi di HIV erano 30,6 milioni con 1.100 .000 bambini (3). Le donne rappresentano il $42 \%$ della popo- lazione infetta e la percentuale è in continuo aumento, poiché ora l'infezione si trasmette con più facilità alle donne piuttosto che agli uomini $(4,5)$.

Le proiezioni per l'anno 2000 parlano di circa 40 milioni di adulti colpiti da HIV, il $90 \%$ dei quali nei paesi in via di sviluppo (6). I dati sono ottenuti considerando, per il 1997, la media di un nuovo caso di infezione ogni cinque secondi: questo significa 10 persone al minuto e 600 all'ora (3). E si pensa che, entro il 2000, il numero di bambini orfani a causa dell' AIDS raggiungerà i 10 milioni $(2,3)$. Nel 1996 i decessi per AIDS segnalati all'Organizzazione Mondiale della Sanità (OMS) erano 1 milione e mezzo, ma probabilmente si trattava di una cifra approssimata per difetto, poiché il sistema di raccolta delle informazioni è ancora piuttosto arretrato in alcuni paesi, specialmente quelli in via di sviluppo.

Dall'inizio della pandemia globale, all'OMS sono stati documentati 8,4 milioni di casi di AIDS e le regioni maggiormente interessate sono l'Africa centrale e sub sahariana, l'America Latina, l'Estremo Oriente, il Nord America e l'Europa occidentale $(2,3)$. Un'esatta valutazione della sua diffusione è al momento impossibile, a Facoltà di Farmacia, Università di Ljubljana, Slovenia $\S$ Director of Outcomes Research \& Management, Merck \& Co., Inc., West Point 
e non si possono conoscere con sicurezza i come:

costi indiretti, i traumi e la perdita di risorse - diretti (ad esempio il costo dei farmaci, dei umane in determinate aree.

Anche se l'AIDS rimane uno dei principali fattori di morte per gli uomini e le donne tra i 25 e 44 anni, per la prima volta nella storia dell'epidemia si assiste a una marcata diminuzione dei decessi tra le persone infette, grazie al rallentare della diffusione e al miglioramento nelle cure (5). Ciononostante, l'AIDS continua a diffondersi nel mondo (7). La prevenzione rimane quindi il rimedio migliore e più efficace, anche dal punto di vista dei costi, per tenere sotto controllo l'AIDS e salvare vite umane.

\section{Trattamento della malattia}

Tre sono gli aspetti del trattamento dell'AIDS che sono fonte di spesa: la diagnostica, la terapia e il follow-up. Si conoscono quattro strategie (terapie) che prolungano la vita dei pazienti: la terapia antiretrovirale, la profilassi per la polmonite da Pneumocystis carinii e da Mycobacterium avium, e la cura presso un medico con esperienza di HIV (9). Negli Stati Uniti sono registrati attualmente 11 agenti antiretrovirali: cinque nucleosidi inibitori della transcrittasi inversa (didanosina, lamivudina, stavudina, zalcitabina e zidovudina), due nonucleosidi inibitori della transcrittasi (delaviridina e nevirapina) e quattro inibitori della proteasi (indinavir, ritonavir, saquina vir e nelfinavir) (10).

Tutti gli agenti retrovirali bloccano differenti stadi del ciclo vitale del virus e sono attualmente usati in numerose terapie, anche se non ci sono prove della maggiore efficacia di una delle combinazioni. La scelta della combinazione da somministrare a un paziente solitamente si basa sulle linee guida della terapia, che comprendono anche l'esperienza del medico curante, lo stato di salute del paziente e gli aspetti economici. Il primo mix di farmaci, composto da zidovudina e lamivudina, è stato registrato nell'ottobre del 1997 con il nome di Combivir $^{\circledR}$.

Studi farmaeconomici sui costi del trattamento dell'AIDS

L'obiettivo principale della farmaeconomia è presentare i risultati delle terapie, dei servizi e dei prodotti nei termini di unità monetarie, in relazione alle risposte cliniche. L'aggiunta di questa variabile, che permette di considerare anche gli aspetti economici, migliora la descrizione sistematica del problema. E se la farmaeconomia ha avuto un notevole sviluppo in questi anni è proprio in conseguenza degli elevati costi del sistema sanitario.

Scopo precipuo delle analisi di farmaeconomia è quindi l'identificazione, la misurazione e la presentazione dei costi. I costi si definiscono

laboratori, dei ricoveri in ospedale, delle visite ambulatoriali);

- indiretti (ad esempio i costi sostenuti dalla

famiglia del malato, come le spese di trasporto; la mancanza di guadagni dovuta a malattia o morte prematura; la perdita di produttività in caso di invalidità);

- intangibili (ad esempio stati di sofferenza, traumi e depressione).

In questa era di contenimento dei costi, si pone una grande enfasi sulla riduzione delle spese per i medicinali, ma il costo non può essere l'unico criterio considerato nella scelta di un farmaco. Bisogna infatti valutare anche l'efficacia e i benefici a lungo termine di una determinata terapia, prima di fare qualsiasi prescrizione. E occorre tener ben presente che, nei casi di infezione da HIV, i costi indiretti (l'impiego previsto, la perdita di produttività, ecc...) sono di gran lunga superiori a tutti gli altri costi.

\section{OBIETTIVI}

Il presente articolo si prefigge di stendere una breve lista dei costi che devono essere inclusi in un modello o in uno studio farmaeconomico sull'infezione da HIV o AIDS.

\section{MATERIALI E METODI}

\section{Fonti}

Gli studi pubblicati tra il gennaio 1995 e l'agosto del 1997 sono stati rivisti, integrando i dati sulla base dei database di Medline, AIDSline e Current Contents. Sono state inserite informazioni reperite da siti web di riferimento e sono stati aggionnati e rivisti anche gli atti degli ultimi tre convegni internazionali sull'AIDS (XI Convegno Internazionale sull'AIDS di Vancouver, luglio 1996; Terzo Congresso sulle Terapie Farmacologiche nell'Infezione da HIV, Birmingham, U.K., novembre 1996; Quarta Conferenza sui Retrovirus e le Infezioni Opportunistiche, Washington, DC, gennaio 1997). Nel caso di studi su farmaci relativamente nuovi, sono state contattate direttamente le aziende produttrici per ottenere informazioni addizionali.

\section{Estrazione e formulazione dei dati}

I dati ottenuti a partire dalla letteratura di riferimento sono stati raccolti ed elaborati secondo un modulo di collegamento che permette di integrare tutti i tipi di costi. 


\section{RISULTATI E DISCUSSIONE}

Studi sui costi diretti

Calcolo dei costi per i farmaci. Di solito si considerano i prezzi medi all'ingrosso. Quando è possibile si dovrebbe far riferimento ai prezzi dei generici equivalenti invece dei prezzi dei farmaci nuovi. Il calcolo dei costi per le monoterapie e per le combinazioni di farmaci si deve basare sulla dose prescritta, considerando anche il numero di unità che il paziente deve assumere ogni giorno. Un'altra importante questione da osservare riguarda la conformità: occorrerebbe sempre, almeno laddove è possibile, tenere presente il consto della non conformità.

Costo di ospedalizzazione. Si può calcolare in base al singolo giorno di degenza oppure secondo un altro modulo appropriato, valutando i servizi offerti dalla struttura ospedaliera e i costi attuali dei singoli servizi.

Costo dei test di laboratorio e di controllo (follow-up). Nel trattamento di una patologia si effettuano innanzitutto i test diagnostici e quindi, se necessario, i test di controllo. La necessità di eseguire certi tipi di test dipende dalle caratteristiche del paziente, dalle sue predisposizioni famigliari e dall'ambiente in cui vive. Un altro parametro importante, che può influenzare molto la composizione dei costi, è la frequenza con la quale un paziente deve sottoporsi a controllo. Solitamente l'analisi del costo dei test si svolge su base annuale, dal momento che un anno sembra un appropriato lasso di tempo di follow-up. Gli esami che vengono comunemente prescritti sono: l'emocromo e il profilo ematochimico; il conteggio dei linfociti (conta dei $\mathrm{CD}_{4}^{+}, \mathrm{CD} \%$, rapporto $\mathrm{CD}_{4}^{+} /$ $\mathrm{CD})$; la valutazione dell' incremento della viremia (HIV-RNA); in sierologia (RPR o VDRL, IgG per il toxoplasma, PPD); una radiografia al torace; il test di Papanicolau; l'esame colturale del secreto cervicale per gonorrea e/o clamidia; l'esame del secreto vaginale; e il controllo dell'epatite (antiHFs e anti-HBc, HBsAg e anti-HCV) $(9,12)$.

Costo delle visite di controllo. Per individuare il costo su base annua, si prende a riferimento la spesa per una singola visita e la si moltiplica per il numero di visite necessarie in un anno, numero che dipende dalla situazione clinica e dallo status immunologico del paziente. Notevole importanza assume in questo caso la scelta del medico, poiché è stato dimostrato che i pazienti regolarmente seguiti da medici con esperienza di HIV hanno conseguito risultati migliori.
Costo del trattamento degli effetti collaterali. Le possibili variazioni nelle spese per la terapia contro gli effetti collaterali si manifestano in modo evidente in gruppi di pazienti suddivisi a seconda della conta delle cellule $\mathrm{CD} 4^{+}$. L'individuazione di questo tipo di costo è un parametro prezioso nella valutazione dei vari stati della malattia e nella definizione delle tempistiche per l'inizio della profilassi contro le diverse infezioni, oltre a essere un valido indicatore nella prevenzione degli effetti collaterali (13).

Costo del trattamento delle infezioni opportunistiche. Il valore di questa variabile si può facilmente ricavare dall' analisi su un gruppo di pazienti, considerando come parametro indicativo dello stato del singolo il conteggio delle cellule $\mathrm{CD} 4^{+}$.

Costo delle cure a domicilio. Questi parametri si possono riunire in una stessa sezione dei costi diretti privati, che comprendono spese extra quali la cura personale, il cibo e i pasti, il mantenimento della casa e il giardinaggio, i trasporti (14):

1. trasporti (stimato come un terzo dei costi privati, comprende anche il mantenimento del veicolo):

- mezzi di trasporto per raggiungere lo studio del medico, il laboratorio analisi, la farmacia, lo specialista di medicina alternativa;

- una persona che accompagni il paziente (un membro della famiglia o una persona di casa); - la perdita di ore di lavoro o anche di un'intera giornata lavorativada parte di almeno due persone.

2. mantenimento della casa:

- il paziente deve pagare qualcuno che si occupi della casa e delle piccole riparazioni;

- il paziente dedito al giardinaggio non può seguire la semina delle piante e la raccolta dei frutti.

3. cura personale

- una buona disponibilità di acqua per prevenire infezioni batteriche;

- integratori dietetici e vitamine;

- pasti regolari e appropriati, con frutta e verdura fresca;

- impossibilità di effettuare acquisti in magazzini e supermarket.

Costo per l'assistenza qualificata/giorni di ricovero. Questa sezione può costituire anche una parte preponderante del costo totale: le infermiere specializzate infatti sono sempre molto richieste negli ospedali e non hanno molto tempo, così i pazienti devono procurarsi infermiere private.

Costo per ulteriori visite specialistiche. Se il paziente non è soddisfatto del proprio status 
o la terapia scelta causa complicazioni, si rendono necessari ulteriori approfondimenti clinici.

Costo delle visite in pronto soccorso. In uno studio completo dovrebbero essere presi in considerazione anche questo tipo di costi.

Alcuni dei parametri indicati possono ottenersi solo da una sperimentazione sulla base di un gruppo di pazienti.

\section{Studi sui costi indiretti}

Impieghi previsti/guadagni. Spesso la malattia costringe pazienti nel pieno della loro carriera a prendere un periodo di congedo (15), quindi bisogna considerare come costi indiretti anche:

- i pazienti che si sono licenziati o che hanno ridotto l'orario di lavoro;

- i pazienti che non possono esercitare la-vori impegnativi;

- la perdita di guadagni per il singolo individuo;

- la perdita di guadagno per le aziende;

- la diminuzione della produttività;

- la necessità di sostituire il posto di lavoro del malato;

- l'impegno di un altro lavoratore per il trasporto del paziente.

Perdita di risorse umane. Costituisce un parametro molto delicato, la cui misurazione è difficilmente realizzabile. In questo studio abbiamo perciò preso in considerazione due esempi: 1. i bambini orfani. Alcune stime dicono che, entro l'anno 2000, ben 10 milioni di bambini perderanno entrambi i genitori o almeno la madre, e perciò diventeranno un serio problema per la società, in quanto se ne dovranno occu- pare altre persone. Inoltre la maggior parte dei casi si dovrebbe verificare nei paesi in via di sviluppo, che non hanno a disposizione fondi per provvedere alle loro esigenze (tranne forse le risorse dovute a fondi internazionali, come l'UNICEF). Questi bambini saranno costretti a vivere in condizioni molto difficili: alcuni moriranno, mentre altri non riceveranno mai alcuna forma di educazione. Nel mondo industrializzato, i governi nazionali si occupano in qualche modo degli orfani, ma la presenza di una famiglia rimane comunque la migliore garanzia per la crescita equilibrata di un bambino. Abbandonati a loro stessi, privi del calore di una famiglia e di valori di riferimento, gli orfani potrebbero essere facilmente assoldati nelle file della delinquenza.

2. i donatori di sangue. In alcune aree geografiche il contagio si è diffuso a tal punto che per gli istituti sanitari nazionali e le banche del sangue è diventato estremamente difficile reperire sangue non infetto. La finestra diagnostica per i donatori infetti da HIV è particolarmente problematica. Tutto ciò significa che prima $o$ poi i donatori di sangue sani verranno a mancare in alcune parti del mondo, e a quel punto la malattia si diffonderà ancora più velocemente.

\section{Costi intangibili}

Questa sezione rappresenta forse quella più complessa da analizzare in uno studio approfondito. Esistono diversi protocolli sulla classificazione dei parametri, ma i risultati rimangono ancora piuttosto confusi. Perciò riportiamo qui di seguito gli elementi che devono essere considerati nel nostro caso.

Discriminazioni (15):

- assicurazioni

\begin{tabular}{|l||l|}
\hline \multicolumn{1}{|c||}{ POSITIVO } & \multicolumn{1}{c|}{ NEGATIVO } \\
\hline - Rivalutazione degli obiettivi e delle priorità & - Mutamenti nello stile di vita del paziente (ad \\
nella vita dei pazienti & esempio, mancanza di tempo per i divertimenti) \\
- Aumento delle attenzioni da parte di & - Cambiamenti nelle abitudini dei famigliari e \\
famigliari, partner e amici & amici (ad esempio, dover somministrare \\
- Maggiore consapevolezza di sé & medicinali nel cuore della notte) \\
& - Variazioni nella relazione con la famiglia \\
& biologica \\
& - Cambio di atteggiamento dei colleghi di \\
& lavoro \\
& - Trasformazione o perdita dei rapporti \\
& d'amicizia \\
& - Evitare le attività sociali per paura di essere \\
& attaccati verbalmente \\
& - Difficoltà nell'acquisto di beni immobili \\
& - Stress \\
& - Depressione (diffusione pari al 4-11\%) (xvi) \\
& - Demenza (diffusione pari al 6,5-7,5\%) (xvi) \\
& - Perdita di dignità e orgoglio \\
\hline
\end{tabular}

Tabella 1

Impatto della malattia (sociale, sessuale) sulla qualità della vita 
- cure dentistiche

- cure mediche

- lavoro

- educazione

- sistemazione in alloggio.

Altri effetti. Stati emozionali negativi (dolore, depressione, ansietà).

I costi descritti sono stati individuati rivedendo la letteratura più recente in campo farmaeconomico. Considerando la totalità dei costi, l'impatto sulla società è sicuramente la conseguenza economica maggiore, dal momento che solitamente le persone affette da HIV sono giovani e nel pieno della vita produttiva. Possiamo quindi affermare che i costi indiretti (impieghi previsti e impatto sulla società) sono di gran lunga maggiori rispetto ai costi per le terapie e il controllo. "La diffusione delle terapie contro l'HIV e il monitoraggio del virus probabilmente causano un aumento nei costi a breve termine, ma devono essere viste nel contesto del mantenimento in salute della popolazione giovane e in età produttiva. Bisogna perciò valutare gli effetti che le terapie hanno sulla riduzione dei costi per i ricoveri in ospedale e per la cura delle malattie opportunistiche e anche, almeno potenzialmente, sul rallentamento della trasmissione dell'HIV" (13).

\section{Costi occasionali}

Esiste un'altra classe di costi che non abbiamo descritto: i costi occasionali. È molto difficile operare una reale misurazione di queste spese, almeno fino a quando non si conosce il destino di un particolare paziente. Tuttavia, in alcuni studi di gruppo, viene considerato anche questo tipo di parametro. Prendiamo ad esempio il caso di un giovane avvocato di 35 anni morto di AIDS: sapendo che nella sua carriera presso lo studio legale XYZ ha vinto 20 cause all'anno e ha contribuito con la cifra di XXX al fatturato annuale della società, potremmo calcolare a quanto ammonta il suo mancato guadagno per i prossimi 25 anni. E in questo caso avremmo solo valutato le conseguenze economiche, tralasciando gli effetti sulla sua famiglia, sui suoi eventuali figli, eccetera

\section{CONSIDERAZIONI FINALI}

L'infezione da HIV è una malattia infettiva mortale, che colpisce specialmente le persone giovani e attive in tutto il mondo. Alla fine del XX secolo, sembra essere la maggiore epidemia letale che abbia mai interessato il mondo civilizzato (17). La progressione della malattia o la morte non dipendono da razza, sesso, abuso di sostanze tossiche, condizione socioeconomica o confini geografici (5).

Si trasmette con più facilità alle donne piuttosto che agli uomini, anche se entrambi i gruppi oggi sono a rischio (5). Il trattamento della malattia è associato a costi molto alti per le terapie, il controllo e le altre attività, anche se i costi indiretti sono in realtà ancora maggiori. Il virus colpisce individui nel pieno della loro maturità e li conduce alla morte in 10-15 anni. La terapia contro l'HIV dura tutta la vita e non è ancora stato sviluppato un vaccino per la prevenzione.

Al momento l'approccio terapeutico è l'unico possibile e, anche se molto caro, mantiene ancora un buon rapporto costo/beneficio, perché ritarda lo sviluppo della malattia, arginando la perdita di produttività e quindi i costi per la società.

Questa ricerca rappresenta solo una breve lista dei calcoli farmaeconomici necessari a individuare il costo del trattamento per l'HIV, e speriamo che possa essere di aiuto ai ricercatori che lavorano con i gruppi di pazienti, per mettere a punto un modello generale.

Per descrivere un panorama più complesso dei costi strutturali che interessano gli individui affetti da HIV occorrerebbero ulteriori approfondimenti.

\section{BIBLIOGRAFIA}

1. Joint United Nations: Programme on HIV/AIDS, URL: http://www.cdc.gov/nchs/hiv_aids/internat.htm. Available from Internet. Accessed 1997 Jan 15.

2. John Hopkins: AIDS Service. HIV/AIDS epidemiology, URL:http://www.hopkins-aids.edu/epidemiology/index_epi.html. Available from Internet. 
3. UNAIDS-WHO: Working Group on Global HIV/AIDS and STD Surveillance, Report on the global HIV/AIDS epidemic. December 1997.

4. Schmidt M.E.: HIV update, VA Med Q 1997;124(2):105-7.

5. Flanigan T.P., Uvin S.C., et al.: Update of HIV and AIDS in North America, Med Health RI 1996;79(5):180-7.

6. WHO-FIP: joint declaration: the role of the pharmacist in the fight against the HIV-AIDS pandemic, Presented at FIP Congress, Vancouver, BC, August/September 1997.

7. Guertler I.: Recent aspects of HIV and HIV-related diseases, Infection 1997;25(2):71-3.

8. URL: http://www.cdc.update.nchstp, Available from Internet. Accessed 1997 Feb.

9. Bartlett J.G.::AIDS Service. Management of HIV infection, Available from Internet. URL: http://www.hopkins-AIDS.edu.

10. Correa de Araujo R.: Human immunodeficiency virus infection. Monograph, USPDI Supplement. July 1997.

11. Davidson A.: FIP list message about pharmacoepidemiology, October 1997.

12. Parra E.O..: Standardized forms to use in management of HIV infection, Am Fam Physician 1997;55:2166-72.

13. Gazzard B.G., Moyle J., et al.: British HIV Association guidelines for antiretro-viral treatment of HIV seropositive individuals, Lancet 1997;349:1086-92.

14. Bowie R.D.: The private cost of HIV/AIDS, NZ Med J 1996;109(1016):51-4.

15. Brian T.: Supplying safe drinking water, cost effectively, to persons living with HIV disease and AIDS, Abstract Mo. C. 1674; XI Conference on HIV AIDS, Van-couver, BC, July 1996.

16. Starace F., Van den Boom F., et al.: Future impact of HIV-associated dementia and depression, Abstract Mo. C. 1575; XI Conference on HIV/AIDS, Vancouver, BC, July 1996.

17. Bartlett J.G.: Update in infectious diseases, Ann Intern Med 1997;127:217-24. 